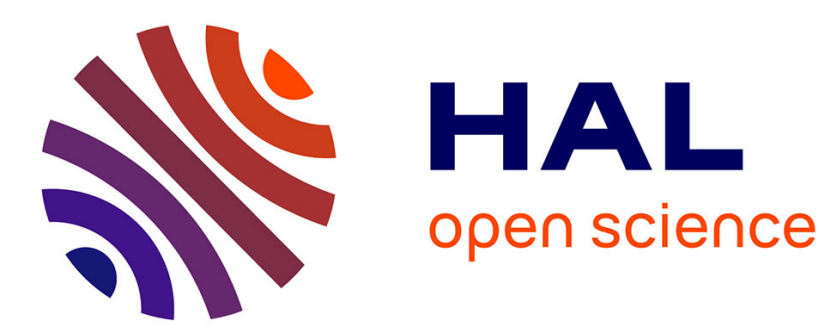

\title{
A simple microstructural viscoelastic model for flowing foams
}

\author{
Rubén Ibáñez, Adrien Scheuer, Emmanuelle Abisset-Chavanne, Francisco
}

Chinesta, Antonio Huerta, Roland Keunings

\section{To cite this version:}

Rubén Ibáñez, Adrien Scheuer, Emmanuelle Abisset-Chavanne, Francisco Chinesta, Antonio Huerta, et al.. A simple microstructural viscoelastic model for flowing foams. International Journal of Material Forming, 2018, 2 (2), pp.295-306. 10.1007/s12289-018-1417-4 . hal-02294107

\section{HAL Id: hal-02294107 https://hal.science/hal-02294107}

Submitted on 23 Sep 2019

HAL is a multi-disciplinary open access archive for the deposit and dissemination of scientific research documents, whether they are published or not. The documents may come from teaching and research institutions in France or abroad, or from public or private research centers.
L'archive ouverte pluridisciplinaire HAL, est destinée au dépôt et à la diffusion de documents scientifiques de niveau recherche, publiés ou non, émanant des établissements d'enseignement et de recherche français ou étrangers, des laboratoires publics ou privés. 


\title{
A simple microstructural viscoelastic model for flowing foams
}

\author{
Rubén Ibáñez ${ }^{1} \cdot$ Adrien Scheuer ${ }^{1,4} \cdot$ Emmanuelle Abisset-Chavanne $^{1} \cdot$ Francisco Chinesta ${ }^{2} \cdot$ Antonio Huerta $^{3}$. \\ Roland Keunings ${ }^{4}$
}

\begin{abstract}
The numerical modelling of forming processes involving the flow of foams requires taking into account the different problem scales. Thus, in industrial applications a macroscopic approach is suitable, whereas the macroscopic flow parameters depend on the cellular structure: cell size, shape, orientation, etc. Moreover, the shape and orientation of the cells are induced by the flow. A fully microscopic description remains useful to understand the foam behaviour and the topological changes induced by the cell elongation or distortion, however, from an industrial point of view, microscopic simulations remain challenging to address practical applications involving flows in complex 3D geometries. In this paper, we propose a viscoelastic flow model where the foam microstructure is represented from suitable microstructure descriptors whose evolution is governed by the macroscopic flow kinematics.
\end{abstract}

Keywords Flowing foams $\cdot$ Viscoelasticity $\cdot$ Conformation $\cdot$ Microstructural description

Francisco Chinesta

Francisco.Chinesta@ensam.eu

Rubén Ibáñez

Ruben.Ibanez-Pinillo@eleves.ec-nantes.fr

Adrien Scheuer

Adrien.Scheuer@uclouvain.be; Adrien.Scheuer@ec-nantes.fr

Emmanuelle Abisset-Chavanne

Emmanuelle.Abisset-Chavanne@ec-nantes.fr

Antonio Huerta

Antonio.Huerta@upc.edu

Roland Keunings

Roland.Keunings@uclouvain.be

1 ICI - High Performance Computing Institute at Ecole Centrale de Nantes, ESI GROUP Chair on Advanced Modeling and Simulation of Manufacturing Processes, 1 rue de la Noe, 44300 Nantes, France

2 PIMM, ENSAM ParisTech, ESI GROUP Chair on Advanced Modeling and Simulation of Manufacturing Processes, 151 Boulevard de l'Hôpital, 75013, Paris, France

3 Laboratori de Càlcul Numèric, Universitat Politècnica de Catalunya, BarcelonaTech, 08034 Barcelona, Spain

4 ICTEAM, Université catholique de Louvain, Av. Georges Lemaitre 4, 1348, Louvain-la-Neuve, Belgium

\section{Introduction}

Aqueous foams are concentrated dispersions of gas bubbles in a surfactant solution. Their structures are organized over a large range of length scales and complex flows take place at different scales [6].

The proposal of macroscopic constitutive equations allows for the efficient modelling and simulation of industrial processes involving the flow of foams $[1,4]$. Usually, such descriptions remain however too phenomenological, and even though they predict accurately the flow kinematics, microstructure information remains often unaccessible. On the opposite side, fully microscopic simulations allow for very detailed descriptions of the foam microstructural evolution [2]. However such approaches fail to address scenarios of industrial interest that usually involve the flow of foams in very large and complex $3 \mathrm{D}$ geometries.

The macroscopic flow model is expected to depend on the cellular structure: cell size, shape and orientation, as well as on the fluid rheology and the surface tension. Moreover, cell shape and orientation are induced by the flow. This microscopic information could be introduced into a macroscopic flow model by using standard upscaling and homogenization techniques. Thus inspired by [7], at some locations in the domain in which an effective homogeneous fluid flows, we could attach a representative 
volume containing several cells, whose size, shape and orientation depend on the considered location. Now, a detailed microscopic calculation could be carried out in order to determine the effective fluid rheology. However, such a route, widely and successfully considered in a variety of fields, remains expensive from a computational point of view despite some attempts at combining it with advanced model reduction techniques [12].

The most appealing description consists of a macroscopic flow model making use of some conformational variables describing the main microstructural features, as widely considered in the field of multiscale polymer modelling [3, 11]. Thus, in [10] the authors study flows during foaming, considering cell evolutions but without addressing the shape orientation and then, the induced anisotropy. Richer microstructure descriptions can be obtained by using a set of configurational coordinates, from which a conformation tensor can be derived and a macroscopic constitutive equation established. This route was successfully considered in [13]. In the present work, we propose an alternative simpler microstructure description and its coupling with the macroscopic flow.

Remark In the sequel, we consider the following tensor products, where Einstein's summation convention is assumed:

- if $\mathbf{a}$ and $\mathbf{b}$ are first-order tensors, the single contraction $\cdot \operatorname{reads}(\mathbf{a} \cdot \mathbf{b})=a_{j} b_{j}$

- if $\mathbf{a}$ and $\mathbf{b}$ are first-order tensors, the dyadic product $\otimes$ reads $(\mathbf{a} \otimes \mathbf{b})_{j k}=a_{j} b_{k}$;

- if $\mathbf{a}$ and $\mathbf{b}$ are respectively second and first-order tensors, the single contraction $\cdot$ reads $(\mathbf{a} \cdot \mathbf{b})_{j}=a_{j m} b_{m}$;

- if $\mathbf{a}$ and $\mathbf{b}$ are second-order tensors, the single contraction $\cdot \operatorname{reads}(\mathbf{a} \cdot \mathbf{b})_{j k}=a_{j m} b_{m k}$;

- if $\mathbf{a}$ and $\mathbf{b}$ are second-order tensors, the double contraction : reads $(\mathbf{a}: \mathbf{b})=a_{j k} b_{k j}$.

\section{Cell conformation}

A very simple description of a $3 \mathrm{D}$ cell consists of a deformable ellipsoid (with constant volume) ranging from the spherical shape to the infinite aspect ratio ellipsoid (rod). Such an ellipsoid could be represented by means of three orthogonal extensible springs, with reference length $2 L^{0}$ and stiffness $\mathcal{K}$. In the sequel, we restrict our analysis to $2 \mathrm{D}$ scenarios (by considering ellipses instead of ellipsoids) but the derived models and their numerical solution procedures can be straightforwardly extended to 3D.

First, we consider the kinematics of a single linear elastic dumbbell as starting point for elaborating the cell conformation.

\section{Kinematics of an extensible rod}

The extensible rod, of reference length $2 L^{0}$ and assumed aligned in direction $\mathbf{p}$ ( $\mathbf{p}$ having a unit norm), is represented by an elastic spring of length $2 L$ (in the deformed state) and stiffness $\mathcal{K}$ equipped with two beads at its extremities where hydrodynamic forces act. In the sequel, the word hydrodynamic refers to the viscous drag force and not to the one considered in other works to describe the effects of a bead kinematics on the others from the use of the Oseen tensor. These forces scale with the fluid - bead relative velocity, the former given by $\mathbf{v}_{0}+\nabla \mathbf{v} \cdot \mathbf{p} L$ and the latter by $\mathbf{v}_{G}+\dot{\mathbf{p}} L+\mathbf{p} \dot{L}$, where $\mathbf{v}_{0}$ is the unperturbed fluid velocity at the rod center of gravity and $\mathbf{v}_{G}$ the velocity of the rod centre of gravity. A sketch of the rod and the forces acting on it is depicted in Fig. 1.

The system is assumed inertialess, that implies the equilibrium of forces and torques. The first implies $\mathbf{F}^{H}(\mathbf{p} \mathbf{L})+\mathbf{F}^{H}(-\mathbf{p} L)=\mathbf{0}$, leading to $\mathbf{v}_{0}=\mathbf{v}_{G}$, that is, the rod centre of gravity moves with the fluid.

Now, to prevent a resultant torque, force $\mathbf{F}^{H}(\mathbf{p} L)$ must align with $\mathbf{p}$, i.e. $\mathbf{F}^{H}(\mathbf{p} L)=\lambda \mathbf{p}, \lambda \in \mathbb{R}$. Thus, we have

$\mathbf{F}^{H}(\mathbf{p} L)=\xi(\nabla \mathbf{v} \cdot \mathbf{p} L-\dot{\mathbf{p}} L-\mathbf{p} \dot{L})=\lambda \mathbf{p}$,

that multiplying by $\mathbf{p}$ and taking into account that $\mathbf{p} \cdot \mathbf{p}=1$ and consequently $\mathbf{p} \cdot \dot{\mathbf{p}}=0$, yields

$\xi(\nabla \mathbf{v}:(\mathbf{p} \otimes \mathbf{p}) L-\dot{L})=\lambda$,

expression that introduced into Eq. 1 reads

$\xi(\nabla \mathbf{v} \cdot \mathbf{p} L-\dot{\mathbf{p}} L-\mathbf{p} \dot{L})=\xi(\nabla \mathbf{v}:(\mathbf{p} \otimes \mathbf{p}) L-\dot{L}) \mathbf{p}$,

that leads to the rotary velocity $\dot{\mathbf{p}}$

$\dot{\mathbf{p}}=\nabla \mathbf{v} \cdot \mathbf{p}-\nabla \mathbf{v}:(\mathbf{p} \otimes \mathbf{p}) \mathbf{p}$,

that is nothing else than the standard Jeffery expression for ellipsoids of infinite aspect ratio (rods) [9].

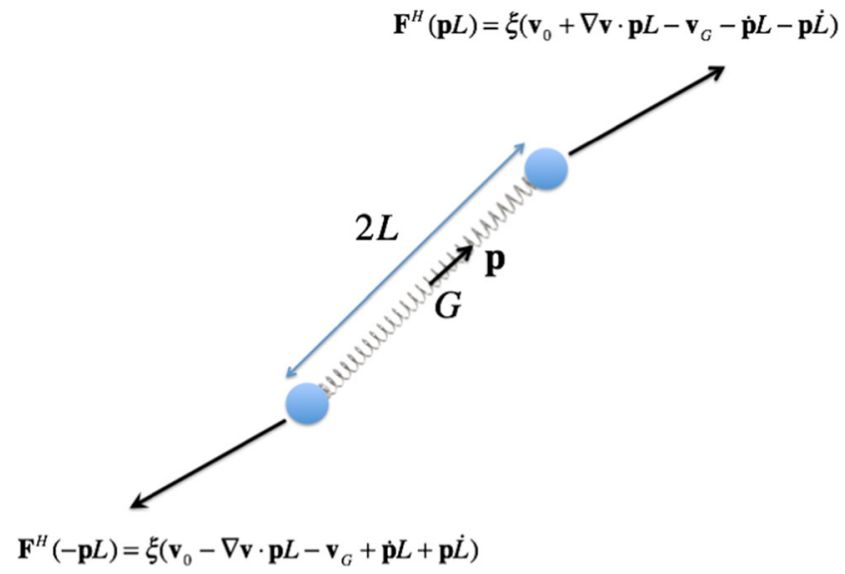

Fig. 1 Extensible rod immersed in a flow 
Now, by equating the force acting on the beads $\lambda$ with the one within the spring, we have

$2 \mathcal{K}\left(L-L^{0}\right)=\xi(\nabla \mathbf{v}:(\mathbf{p} \otimes \mathbf{p}) L-\dot{L})$,

or

$\left.\dot{L}=-\frac{2 \mathcal{K}}{\xi}\left(L-L^{0}\right)+\nabla \mathbf{v}:(\mathbf{p} \otimes \mathbf{p}) L\right)$.

Thus, the kinematics of an elastic dumbbell of reference length $2 L^{0}$ with conformation at time $t$ given by its orientation $\mathbf{p}$ and length $2 L$, read

$$
\left\{\begin{array}{l}
\dot{\mathbf{p}}=\nabla \mathbf{v} \cdot \mathbf{p}-\nabla \mathbf{v}:(\mathbf{p} \otimes \mathbf{p}) \mathbf{p} \\
\left.\dot{L}=-\frac{2 \mathcal{K}}{\xi}\left(L-L^{0}\right)+\nabla \mathbf{v}:(\mathbf{p} \otimes \mathbf{p}) L\right)
\end{array} .\right.
$$

\section{From rigid ellipses to orthogonal elastic bi-dumbells}

In [5] it was proven that in order to represent a rigid ellipse whose kinematics are given by the Jeffery equation it suffices to consider a rigid system composed of two mutually orthogonal rods whose lengths correspond with the length of the ellipse axes.

In this case, if $\mathbf{p}$ refers to the direction of the ellipse largest axis, and $\mathcal{F}=\frac{r^{2}-1}{r^{2}+1}$, with $r$ the ellipse aspect ratio, we have

$\dot{\mathbf{p}}=\mathbf{\Omega} \cdot \mathbf{p}+\mathcal{F}(\mathbf{D} \cdot \mathbf{p}-\mathbf{D}:(\mathbf{p} \otimes \mathbf{p}) \mathbf{p})$,

where $\boldsymbol{\Omega}$ and $\mathbf{D}$ are respectively the vorticity and the rate of strain tensors, $2 \boldsymbol{\Omega}=\nabla \mathbf{v}-(\nabla \mathbf{v})^{T}$ and $2 \mathbf{D}=\nabla \mathbf{v}+(\nabla \mathbf{v})^{T}$.

Now, we address the more general case in which both rigid rods are replaced by two extensible and mutually perpendicular springs of reference lengths $L_{1}^{0}$ and $L_{2}^{0}$. In the sequel, the same reference lengths are assumed for both dumbbells, i.e. $2 L_{1}^{0}=2 L_{2}^{0}=2 L^{0}$.

In the present configuration, and considering that as proven in our former works the centre of gravity moves with the fluid, the hydrodynamic forces applying at beads $L_{1} \mathbf{p}_{1}$ and $L_{2} \mathbf{p}_{2}, \mathbf{F}_{1}^{H}$ and $\mathbf{F}_{2}^{H}$ read respectively

$\mathbf{F}_{1}^{H}=\xi\left(\nabla \mathbf{v} \cdot \mathbf{p}_{1} L_{1}-\dot{\mathbf{p}}_{1} L_{1}-\mathbf{p}_{1} \dot{L}_{1}\right)$,

and

$\mathbf{F}_{2}^{H}=\xi\left(\nabla \mathbf{v} \cdot \mathbf{p}_{2} L_{2}-\dot{\mathbf{p}}_{2} L_{2}-\mathbf{p}_{2} \dot{L}_{2}\right)$,

with $\mathbf{p}_{1} \perp \mathbf{p}_{2}$, and with their orientation rates of change expressed from

$\left\{\begin{array}{l}\dot{\mathbf{p}}_{1}=\omega \times \mathbf{p}_{1} \\ \dot{\mathbf{p}}_{2}=\omega \times \mathbf{p}_{2}\end{array}\right.$

The angular momentum balance implies now

$L_{1}^{2} \mathbf{p}_{1} \times\left(\nabla \mathbf{v} \cdot \mathbf{p}_{1}-\dot{\mathbf{p}}_{1}\right)+L_{2}^{2} \mathbf{p}_{2} \times\left(\nabla \mathbf{v} \cdot \mathbf{p}_{2}-\dot{\mathbf{p}}_{2}\right)=\mathbf{0}$,

which coincides with the expression obtained in the case of rigid rods [5], proving the validity of the Jeffery equation in the case of orthogonal elastic bi-dumbells.
Introducing the Jeffery Eq. 8 with $r=\frac{L_{1}}{L_{2}}$ that implies $\mathcal{F}=\frac{L_{1}^{2}-L_{2}^{2}}{L_{1}^{2}+L_{2}^{2}}$, i.e.

$\dot{\mathbf{p}}_{1}=\boldsymbol{\Omega} \cdot \mathbf{p}_{1}+\mathcal{F}\left(\mathbf{D} \cdot \mathbf{p}_{1}-\left(\mathbf{p}_{1}^{T} \cdot \mathbf{D} \cdot \mathbf{p}_{1}\right) \mathbf{p}_{1}\right)$,

into the expression of the hydrodynamic force acting on bead $\mathbf{p}_{1} L_{1}$, we have

$$
\begin{aligned}
\mathbf{F}_{1}^{H}= & \xi\left(\nabla \mathbf{v} \cdot \mathbf{p}_{1} L_{1}-\dot{\mathbf{p}}_{1} L_{1}-\mathbf{p}_{1} \dot{L}_{1}\right) \\
= & \xi\left(\nabla \mathbf{v} \cdot \mathbf{p}_{1} L_{1}-\mathbf{\Omega} \cdot \mathbf{p}_{1} L_{1}\right. \\
& \left.-\mathcal{F}\left(\mathbf{D} \cdot \mathbf{p}_{1} L_{1}-\left(\mathbf{p}_{1}^{T} \cdot \mathbf{D} \cdot \mathbf{p}_{1}\right) \mathbf{p}_{1} L_{1}\right)-\mathbf{p}_{1} \dot{L}_{1}\right) \\
= & \xi L_{1}\left((1-\mathcal{F}) \mathbf{D} \cdot \mathbf{p}_{1}+\mathcal{F}\left(\mathbf{p}_{1}^{T} \cdot \mathbf{D} \cdot \mathbf{p}_{1}\right) \mathbf{p}_{1}\right)-\xi \mathbf{p}_{1} \dot{L}_{1} .
\end{aligned}
$$

\section{Modelling incompressible ellipses from orthogonal elastic bi-dumbells}

The projection of force $\mathbf{F}_{1}^{H}$ in the direction $\mathbf{p}_{1}$ is the one that causes the spring extension, i.e.

$2 \mathcal{K}\left(L_{1}-L^{0}\right)-F_{1}^{I}=\xi L_{1} \mathbf{p}_{1}^{T} \cdot \mathbf{D} \cdot \mathbf{p}_{1}-\xi \dot{L}_{1}$,

where the force $F_{1}^{I}$ ensures the incompressibility constraint. Similar calculations lead to

$2 \mathcal{K}\left(L_{2}-L^{0}\right)-F_{2}^{I}=\xi L_{2} \mathbf{p}_{2}^{T} \cdot \mathbf{D} \cdot \mathbf{p}_{2}-\xi \dot{L}_{2}$,

where it was assumed that $\mathcal{K}_{1}=\mathcal{K}_{2}=\mathcal{K}$.

Thus, the extension velocities read

$\dot{L_{1}}=-\frac{2 \mathcal{K}}{\xi}\left(L_{1}-L^{0}\right)+L_{1} \mathbf{p}_{1}^{T} \cdot \mathbf{D} \cdot \mathbf{p}_{1}+\frac{1}{\xi} F_{1}^{I}$,

and

$\dot{L_{2}}=-\frac{2 \mathcal{K}}{\xi}\left(L_{2}-L^{0}\right)+L_{2} \mathbf{p}_{2}^{T} \cdot \mathbf{D} \cdot \mathbf{p}_{2}+\frac{1}{\xi} F_{2}^{I}$.

Taking into account

$\left\{\begin{array}{l}\mathbf{p}_{1}^{T} \cdot \mathbf{D} \cdot \mathbf{p}_{1}=\mathbf{D}:\left(\mathbf{p}_{1} \otimes \mathbf{p}_{1}\right) \\ \mathbf{p}_{2}^{T} \cdot \mathbf{D} \cdot \mathbf{p}_{2}=\mathbf{D}:\left(\mathbf{p}_{2} \otimes \mathbf{p}_{2}\right)\end{array}\right.$,

and the fact that, since $\mathbf{p}_{1}$ and $\mathbf{p}_{2}$ are mutually orthogonal, $\left(\mathbf{p}_{1} \otimes \mathbf{p}_{1}\right)+\left(\mathbf{p}_{2} \otimes \mathbf{p}_{2}\right)=\mathbf{I}$ (with $\mathbf{I}$ the identity tensor), the incompressibility constraint $\frac{d}{d t}\left(L_{1} L_{2}\right)=0$ reads

$$
\begin{aligned}
\dot{L}_{1} L_{2}+L_{1} \dot{L}_{2}= & -\frac{2 \mathcal{K}}{\xi}\left(L_{1}-L^{0}\right) L_{2}-\frac{2 \mathcal{K}}{\xi}\left(L_{2}-L^{0}\right) L_{1} \\
& +F_{1}^{I} \frac{L_{2}}{\xi}+F_{2}^{I} \frac{L_{1}}{\xi}=0
\end{aligned}
$$

which simplifies to

$$
\begin{aligned}
\dot{L}_{1} L_{2}+L_{1} \dot{L}_{2}= & -\frac{4 \mathcal{K}}{\xi} L_{1} L_{2}+\frac{2 \mathcal{K}}{\xi} L^{0}\left(L_{1}+L_{2}\right) \\
& +F_{1}^{I} \frac{L_{2}}{\xi}+F_{2}^{I} \frac{L_{1}}{\xi}=0 .
\end{aligned}
$$

Forces related to the incompressibility constraint are expected to contribute isotropically to the resulting macroscopic stress, and then taken in the flow incompressibility 
constraint. Thus, if we compute the contribution of $F_{1}^{I}$ and $F_{2}^{I}$ to the stress by using the Kramers rule, we have

$$
\begin{aligned}
\boldsymbol{\sigma}^{I} & =F_{1}^{I} \mathbf{p}_{1} \otimes L_{1} \mathbf{p}_{1}+F_{2}^{I} \mathbf{p}_{2} \otimes L_{2} \mathbf{p}_{2} \\
& =F_{1}^{I} L_{1}\left(\mathbf{p}_{1} \otimes \mathbf{p}_{1}\right)+F_{2}^{I} L_{2}\left(\mathbf{p}_{2} \otimes \mathbf{p}_{2}\right),
\end{aligned}
$$

which suggests considering $F_{1}^{I} L_{1}=F_{2}^{I} L_{2}$, since $\mathbf{p}_{1} \otimes \mathbf{p}_{1}+$ $\mathbf{p}_{2} \otimes \mathbf{p}_{2}=\mathbf{I}$.

Thus, considering $F_{2}^{I}=F_{1}^{I} \frac{L_{1}}{L_{2}}$ in Eq. 23, we have

$$
F_{1}^{I}\left(\frac{L_{1}^{2}+L_{2}^{2}}{\xi L_{2}}\right)=\frac{4 \mathcal{K}}{\xi} L_{1} L_{2}-\frac{2 \mathcal{K}}{\xi} L^{0}\left(L_{1}+L_{2}\right),
$$

or

$F_{1}^{I}=4 \mathcal{K} \frac{L_{1} L_{2}^{2}}{L_{1}^{2}+L_{2}^{2}}-2 \mathcal{K} \frac{L^{0}\left(L_{1}+L_{2}\right) L_{2}}{L_{1}^{2}+L_{2}^{2}}$,

that vanishes for the relaxed case $L_{1}=L_{2}=L^{0}$, i.e. $F_{1}^{I}\left(L_{1}=L_{2}=L^{0}\right)=0$ and consequently $F_{2}^{I}\left(L_{1}=L_{2}=\right.$ $\left.L^{0}\right)=0$.

Thus, finally the governing equations for the orthogonal elastic bi-dumbbell representing an incompressible ellipse read:

$$
\left\{\begin{array}{l}
\dot{\mathbf{p}}_{1}=\boldsymbol{\Omega} \cdot \mathbf{p}_{1}+\frac{L_{1}^{2}-L_{2}^{2}}{L_{1}^{2}+L_{2}^{2}} \mathbf{D} \cdot \mathbf{p}_{1}-\frac{L_{1}^{2}-L_{2}^{2}}{L_{1}^{2}+L_{2}^{2}}\left(\mathbf{p}_{1}^{T} \cdot \mathbf{D} \cdot \mathbf{p}_{1}\right) \mathbf{p}_{1} \\
\dot{L}_{1}=-\frac{2 \mathcal{K}}{\xi}\left(L_{1}-L^{0}\right)-\frac{1}{\xi} F_{1}^{I}+L_{1} \mathbf{p}_{1}^{T} \cdot \mathbf{D} \cdot \mathbf{p}_{1} \\
\dot{L}_{2}=-\frac{L_{2}}{L_{1}} \dot{L}_{1} \\
F_{1}^{I}=4 \mathcal{K} \frac{L_{1} L_{2}^{2}}{L_{1}^{2}+L_{2}^{2}}-2 \mathcal{K} \frac{L^{0}\left(L_{1}+L_{2}\right) L_{2}}{L_{1}^{2}+L_{2}^{2}} \\
F_{2}^{I}=F_{1}^{I} \frac{L_{1}}{L_{2}}
\end{array}\right.
$$

\section{Conformation descriptor}

When considering control volumes (small enough with respect to the flow but large enough with respect to the cell size) in a flowing foam, it can be observed that the cells in each control volume have similar shapes and orientations. In that case, the cell population in each volume element can be described using $\mathbf{p}_{1}$ and $L_{1}$ (when $L^{0}$ is assumed known).

The contribution of a cell to the stress using the Kramers rule can be obtained from the elastic forces. The contribution due to the incompressibility constraint being isotropic, it can be aggregated to the pressure term. Thus, the conformation contribution $\sigma^{c}$ results

$$
\begin{aligned}
\sigma^{c} & =2 \mathcal{K}\left(L_{1}-L^{0}\right) \mathbf{p}_{1} \otimes L_{1} \mathbf{p}_{1}+2 \mathcal{K}\left(L_{2}-L^{0}\right) \mathbf{p}_{2} \otimes L_{2} \mathbf{p}_{2} \\
& =2 \mathcal{K}\left(L_{1}-L^{0}\right) L_{1}\left(\mathbf{p}_{1} \otimes \mathbf{p}_{1}\right)+2 \mathcal{K}\left(L_{2}-L^{0}\right) L_{2}\left(\mathbf{p}_{2} \otimes \mathbf{p}_{2}\right) \\
& =2 \mathcal{K}\left(\Delta L_{1} L_{1}\left(\mathbf{p}_{1} \otimes \mathbf{p}_{1}\right)+\Delta L_{2} L_{2}\left(\mathbf{p}_{2} \otimes \mathbf{p}_{2}\right)\right),
\end{aligned}
$$

that vanishes in the relaxed configuration $L_{1}=L_{2}=L^{0}$, with $\Delta L_{1}=0$ and $\Delta L_{2}=0$.
In these circumstances, the simplest choice for the conformation tensor consists of the second-order symmetric tensor $\mathbf{c}$ defined from

$\mathbf{c}=\Delta L_{1} L_{1}\left(\mathbf{p}_{1} \otimes \mathbf{p}_{1}\right)+\Delta L_{2} L_{2}\left(\mathbf{p}_{2} \otimes \mathbf{p}_{2}\right)$.

It is important to note that the conformation does not involve an averaging process affecting the different cells inside the control volume because we assumed that all of them share almost the same conformation. However, as it is usual in the modelling of suspensions, this hypothesis could easily be relaxed and we would thus consider the average of different cell conformation, as addressed in the micromacro simulations by Keunings and coworkers using the Lagrangian Particle Method - LPM - [8, 11].

Even though many rheological behaviours could be associated to flowing foams, this paper focuses on the consideration of induced anisotropy due to the cell deformation. For this reason, and without loss of generality, we consider in what follows the simplest rheological behaviour consisting of a Newtonian behaviour complemented with an elastic contribution related to the cell deformation. In any case, more complex bulk rheologies could be considered, as for example the one related to a viscoplastic behaviour.

Thus, the macroscopic viscoelastic constitutive equation reads

$\boldsymbol{\sigma}=-p \mathbf{I}+2 \eta \mathbf{D}+\mu \mathbf{c}$,

where $p$ is the pressure, that can be viewed as the Lagrange multiplier associated with the macroscopic flow incompressibility constraints, $\eta$ the effective homogenized fluid viscosity, and $\mu$ the rheological parameter affecting the contribution of the microscopic conformation that scales with $\mathcal{K}$ and the volume concentration of cells.

The origin of the elasticity introduced into the conformation field evolution is related to the surface tension that resists the cell deformation from its spherical reference conformation. Topological changes can operate at the elemental cell level. However, on average their net effect is to avoid too large cellular distorsions. Thus, the coefficient $\mu$ should describe both the surface tension as well as the microscopic topological changes. In the proposed model, it remains purely phenomenological and should be identified from appropriate rheological tests.

Even though the present model does not address relaxation mechanisms, they could easily be incorporated by including a viscous component at the spring-beads level.

\section{Rheological behaviour}

In order to study the rheological response of the proposed model, we carried out a numerical study to obtain the 
loss and storage moduli of a foam. To proceed, consider a periodic shear strain

$\gamma(t)=\gamma_{0} \sin (\omega t)$

whose time derivative provides the evolution of the shear rate

$\dot{\gamma}(t)=\gamma_{0} \omega \cos (\omega t)$.

In simple shear flow, the velocity gradient thus reads $\nabla \mathbf{v}=$ $\left[\begin{array}{ll}0 & \dot{\gamma}(t) \\ 0 & 0\end{array}\right]$, and we could use the model Eq. 27 to obtain the time evolution of the shape and orientation of a cell subject to such an oscillatory flow. We can thus obtain the time evolution $\mathbf{c}(t)$ of the conformation tensor introduced in the previous section.

We can now decompose the off-diagonal component of tensor $\mathbf{c}(t)$, that is $c_{12}(t)$ in its in-phase and out-of-phase contributions according to

$c_{12}(t)=c_{12}^{\prime} \sin (\omega t)+c_{12}^{\prime \prime} \cos (\omega t)$,

where $c_{12}^{\prime}$ and $c_{12}^{\prime \prime}$ are analogous to the so-called storage $\left(G^{\prime}\right)$ and loss $\left(G^{\prime \prime}\right)$ modulus respectively (they actually correspond to the storage and loss moduli associated with the stress contribution $\sigma^{c}$ ).

Figure 2 shows the value of $c_{12}^{\prime}$ and $c_{12}^{\prime \prime}$ for an oscillation angular frequency ranging from 0.1 to $100 \mathrm{rad} / \mathrm{s}$. The parameters are $\xi=0.1, \mathcal{K}=1, L^{0}=0.005$ (metric system of units). We can recognize a Maxwell-like model, with the classical slopes of $1\left(G^{\prime \prime}\right)$ and $2\left(G^{\prime}\right)$ at low frequency.
To obtain properly the storage $\left(G^{\prime}\right)$ and loss $\left(G^{\prime \prime}\right)$ moduli, we should also consider the viscous component of the stress tensor. Thus the moduli are given by

$$
\begin{aligned}
G^{\prime} & =\frac{\sigma_{12}^{\prime}}{\gamma_{0}}=\mu \frac{c_{12}^{\prime}}{\gamma_{0}} \\
G^{\prime \prime} & =\frac{\sigma_{12}^{\prime \prime}}{\gamma_{0}}=2 \eta \omega+\mu \frac{c_{12}^{\prime \prime}}{\gamma_{0}} .
\end{aligned}
$$

Finally, we also conducted another rheological test to observe the behaviour of the conformation tensor when a constant shear flow is brutally stopped. As expected, we obtain an exponential relaxation of the tensor components towards zero.

\section{Macroscopic flow problem}

Neglecting inertia, the macroscopic flow problem is defined as follows:

$$
\left\{\begin{array}{l}
\nabla \cdot \boldsymbol{\sigma}=\mathbf{0} \\
\boldsymbol{\sigma}=-p \mathbf{I}+2 \eta \mathbf{D}+\mu \mathbf{c}, \\
\nabla \cdot \mathbf{v}=0
\end{array},\right.
$$

where $\sigma$ is the Cauchy's stress tensor, I the unit tensor and $\mathbf{v}$ the macroscopic fluid velocity field. It can be noticed that the problem reduces to the standard Stokes flow model as soon as $\mu=0$. Incompressibility is justified by the fact that drainage is neglected.

The flow model must be complemented with the conformation expression

$\mathbf{c}=\Delta L_{1} L_{1}\left(\mathbf{p}_{1} \otimes \mathbf{p}_{1}\right)+\Delta L_{2} L_{2}\left(\mathbf{p}_{2} \otimes \mathbf{p}_{2}\right)$,
Fig. 2 Oscillatory shear $-c_{12}^{\prime}$ and $c_{12}^{\prime \prime}$ components

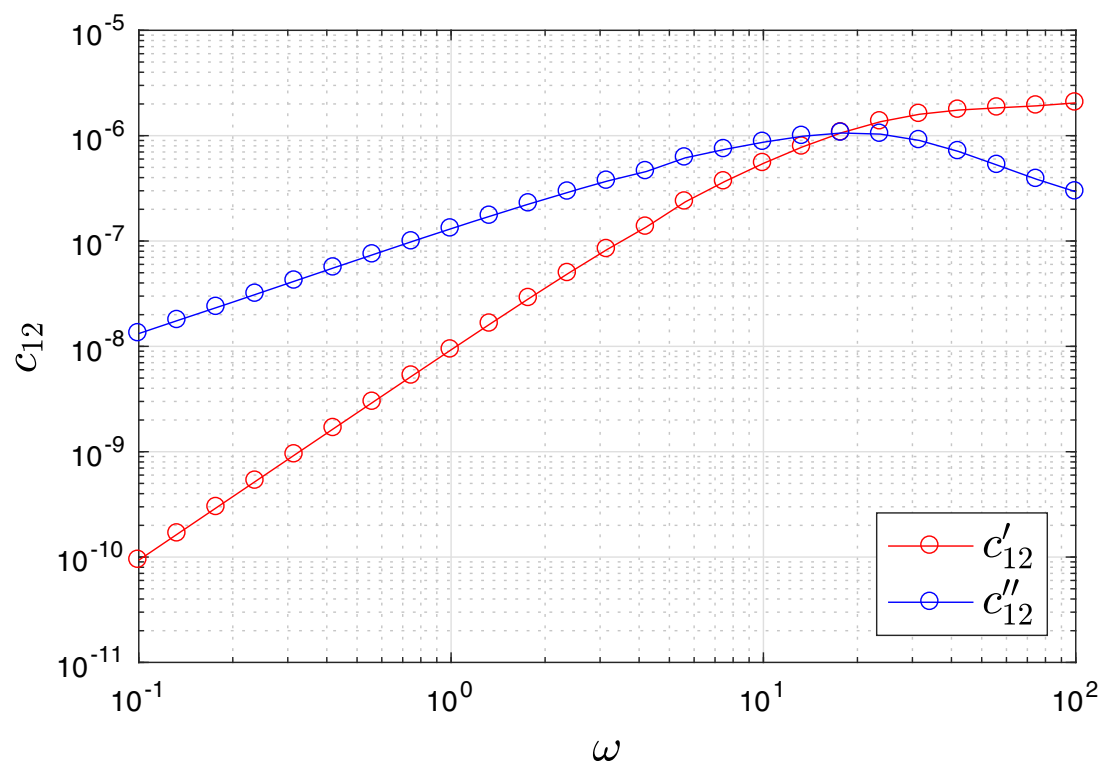


whose evolution is governed by the microstructural model

$$
\left\{\begin{array}{l}
\dot{\mathbf{p}}_{1}=\boldsymbol{\Omega} \cdot \mathbf{p}_{1}+\frac{L_{1}^{2}-L_{2}^{2}}{L_{1}^{2}+L_{2}^{2}} \mathbf{D} \cdot \mathbf{p}_{1}-\frac{L_{1}^{2}-L_{2}^{2}}{L_{1}^{2}+L_{2}^{2}}\left(\mathbf{p}_{1}^{T} \cdot \mathbf{D} \cdot \mathbf{p}_{1}\right) \mathbf{p}_{1} \\
\dot{L}_{1}=-\frac{2 \mathcal{K}}{\xi}\left(L_{1}-L^{0}\right)-\frac{1}{\xi} F^{I}+L_{1} \mathbf{p}_{1}^{T} \cdot \mathbf{D} \cdot \mathbf{p}_{1} \\
\dot{L}_{2}=-\frac{L_{2}}{L_{1}} \dot{L}_{1} \\
F_{1}^{I}=4 \mathcal{K} \frac{L_{1} L_{2}^{2}}{L_{1}^{2}+L_{2}^{2}}-2 \mathcal{K} \frac{L^{0}\left(L_{1}+L_{2}\right) L_{2}}{L_{1}^{2}+L_{2}^{2}} \\
F_{2}^{I}=F_{1}^{I} \frac{L_{1}}{L_{2}}
\end{array}\right.
$$

In order to solve the resulting flow model defined in $\Omega \subset$ $\mathbb{R}^{2}$, appropriate boundary conditions must be enforced at the domain boundary $\Gamma \equiv \partial \Omega$

$\left\{\begin{array}{ll}\mathbf{v}=\mathbf{v}_{g} & \text { in } \Gamma_{D} \\ \mathbf{t}=\boldsymbol{\sigma} \cdot \mathbf{n}=\mathbf{t}_{g} & \text { in } \Gamma_{N}\end{array}\right.$,

with $\Gamma_{D} \cup \Gamma_{N}=\Gamma$ and $\Gamma_{D} \cap \Gamma_{N}=\emptyset$.

\section{Numerical solution strategy}

Cells are assumed represented at the initial time by a spherical conformation tensor $\mathbf{c}=\mathbf{0}$, since the cells are initially in the relaxed state, $L_{1}=L_{2}=L^{0}$.

1. The associated Stokes problem is solved at time $t_{n}$, from the microstructural term given at the previous time step $\mu \mathbf{c}\left(t_{n-1}\right)$. The problem is solved using a standard mixed velocity-pressure formulation using any finite element satisfying the stability conditions, the so-called LBB conditions. In the example reported later, we considered Q9/Q4 finite elements;

2. Then, from the just computed velocity field $\mathbf{v}\left(t_{n}\right)$ the orientation and extension fields, $\mathbf{p}_{1}\left(\mathbf{x}, t_{n}\right)$ and $L_{1}\left(\mathbf{x}, t_{n}\right)$ respectively, are updated by using a first-order discontinuous Galerkin formulation;

3. Finally, the conformation tensor is updated $\mathbf{c}\left(t_{n}\right)$.

These three steps are repeated until reaching the maximum simulation time or the steady state.

\section{Updating the conformation field}

In the numerical experiments described below, we consider 2D flows defined in 2D geometries $\Omega \subset \mathbb{R}^{2}$. In the $2 \mathrm{D}$ case, the unit vector $\mathbf{p}_{1}$ can be expressed by $\mathbf{p}_{1}=$ $(\cos \theta, \sin \theta)^{T}$. By taking the time derivative, we get $\dot{\mathbf{p}}_{1}=$ $\dot{\theta}(-\sin \theta, \cos \theta)^{T}$. Using $\theta$ as orientation descriptor, the Jeffery equation can be expressed as

$\dot{\theta}(-\sin \theta, \cos \theta)^{T}=\mathbf{G}\left(\theta, \nabla \mathbf{v}, L_{1}\right)$.

Multiplying the previous expression by $(-\sin \theta, \cos \theta)$, we obtain the scalar equation

$\dot{\theta}=\mathcal{G}\left(\theta, \nabla \mathbf{v}, L_{1}\right)$, where the material derivative can be expressed in an Eulerian framework by introducing the orientation field $\theta(\mathbf{x}, t)$ whose evolution is governed by

$\frac{\partial \theta}{\partial t}+\mathbf{v} \cdot \nabla \theta=\mathcal{G}\left(\theta, \nabla \mathbf{v}, L_{1}\right)$

The scalar equation governing the evolution of the spring length can also be written as

$\frac{\partial L_{1}}{\partial t}+\mathbf{v} \cdot \nabla L_{1}=\mathcal{H}\left(\theta, \nabla \mathbf{v}, L_{1}\right)$.

Both Eqs. 44 and 45 are purely advective, and thus appropriate discretization taking into account their hyperbolic character must be used. We make here the simplest choice, a first-order discontinuous Galerkin scheme, that considers the generic variable $\mathcal{P}\left(\theta\right.$ or $\left.L_{1}\right)$ constant in each element $\Omega^{e}$ belonging to the mesh $\mathcal{M}$ of $\Omega$. The test function is assumed also constant in each element, vanishing outside. The balance in element $\Omega^{e}$ reads, taking into account the flow incompressibility $(\nabla \cdot \mathbf{v}=0)$ :

$\int_{\Omega^{e}} \frac{\partial \mathcal{P}}{\partial t} d \mathbf{x}+\int_{\Omega^{e}} \nabla \cdot(\mathbf{v} \mathcal{P}) d \mathbf{x}=\int_{\Omega^{e}} \mathcal{J} d \mathbf{x}$,

where the source term $\mathcal{J}$ represents $\mathcal{G}$ or $\mathcal{H}$ depending on the considered equation.

Using the divergence theorem, the second term of the left-hand side can be written from the boundary flux, i.e.

$\int_{\Omega^{e}} \frac{\partial \mathcal{P}}{\partial t} d \mathbf{x}+\int_{\partial \Omega^{e}} \mathcal{P} \mathbf{v} \cdot \mathbf{n} d \mathbf{x}=\int_{\Omega^{e}} \mathcal{J} d \mathbf{x}$,

where $\mathbf{n}$ is the unit outward vector normal to the element boundary. As $\mathcal{P}$ is not defined on $\partial \Omega^{e}$, we consider the element boundary decomposition $\partial \Omega^{e}=\partial^{+} \Omega^{e} \cup \partial^{-} \Omega^{e}$, where $\partial^{-} \Omega^{e}$ and $\partial^{+} \Omega^{e}$ represents the inflow and outflow element boundaries, both defined from $\mathbf{v} \cdot \mathbf{n}<0$ and $\mathbf{v} \cdot \mathbf{n}>0$ respectively. Then we assume that the property on the inflow boundary is given by its value at the upstream element, i.e. $\mathcal{P}\left(\mathbf{x} \in \partial^{-} \Omega^{e}\right)=\mathcal{P}^{e^{-}}$, whereas on the outflow element boundary, it is given by the property at element $\Omega^{e}$, i.e. $\mathcal{P}\left(\mathbf{x} \in \partial^{+} \Omega^{e}\right)=\mathcal{P}^{e}$.

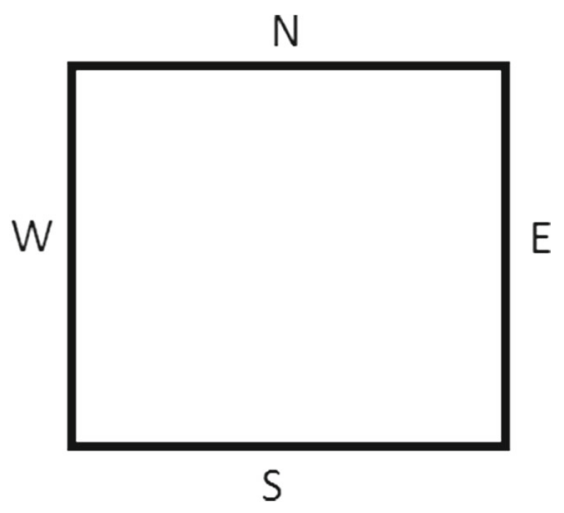

Fig. 3 2D domain 


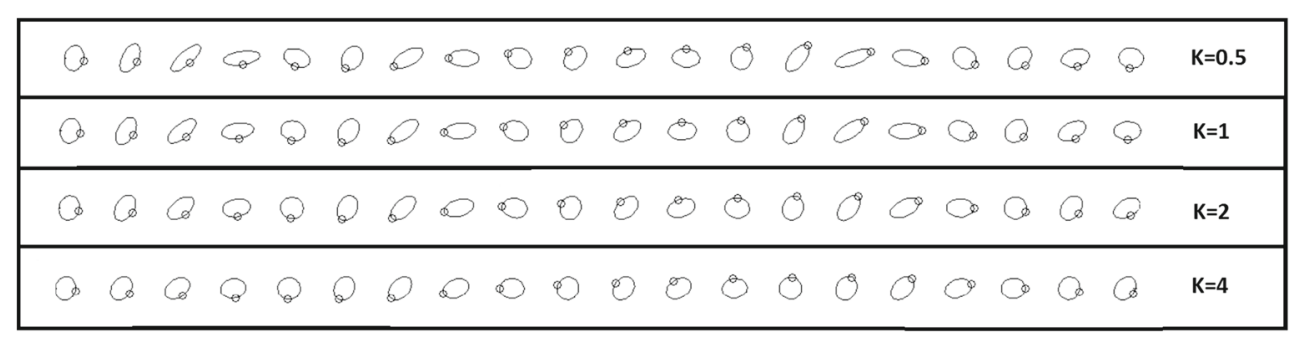

Fig. 4 Conformation evolution for different spring stiffness in a simple shear flow

\section{Discussion}

- In the flow model just proposed there is no size effects, which implies that the characteristic length of cells must be small in relation to the one characterizing the spatial variation of the macroscopic velocity field.

- The mesh considered for integrating the velocity field must be small enough to capture all the macroscopic velocity field details.

- The mesh considered for calculating the conformation field must be small enough for assuming cells in each element of the mesh described by the conformation field inside the element, and it must be large enough for assuming that it represents a population of cells. However, as soon as the model is described in a continuous way, there is no contradiction with the fact of considering elements smaller that the characteristic size of the cell (in the case of polymer flows one can consider elements smaller that the size of a molecule).

\section{Numerical results}

\section{Uncoupled microstructure-flow calculations}

In this section, we address some simple flows in order to evaluate the response of the cells. The model is uncoupled in the sense that the flow induces cell deformation, but the cell conformation does not affect the flow kinematics. Thus, even though some of the flows addressed here could exhibit rich kinematics in practice, in what follows the uncoupled solution does not allow to capture such a rich kinematics, as for example shear banding. Moreover, the only interest of this section being the evolution of the cell deformation and its macroscopic description, rheology is not considered in the analyses carried out.

In the numerical examples considered in this section a unit square is considered as depicted in Fig. 3. The initial (relaxed) conformation is given by a zero conformation tensor $\mathbf{c}(\mathbf{x}, t=0)=\mathbf{0}$.

Four different flows are considered: (i) a simple shear; (ii) a contraction flow; (iii) the driven cavity flow problem and finally (iv) the flow around a square obstacle. In all cases, the microstructure was computed on the basis of the associated Stokes kinematics, i.e. considering the uncoupled flow associated with $\mu=0$.

In order to quantify the way the flow behaviour (shear, rigid motion or elongation) affects the microstructure evolution we consider a flow criterion. For that purpose, first, we introduce the relative rate of rotation $\mathbf{W}$ from

$\mathbf{W}=\omega-\mathbf{w}$,

where $\omega$ is related to the flow vorticity $\Omega$ according to

$\boldsymbol{\Omega}=\boldsymbol{\epsilon} \cdot \boldsymbol{\omega}$,

where $\epsilon$ is the third-order permutation tensor (also known as the Levi-Civita tensor). The vector $\boldsymbol{\omega}$ can also be written in terms of the curl of the velocity,

$\omega=-\frac{1}{2} \nabla \times \mathbf{v}$
Fig. 5 Velocity field (left) and flow criterion (right) in a simple shear flow
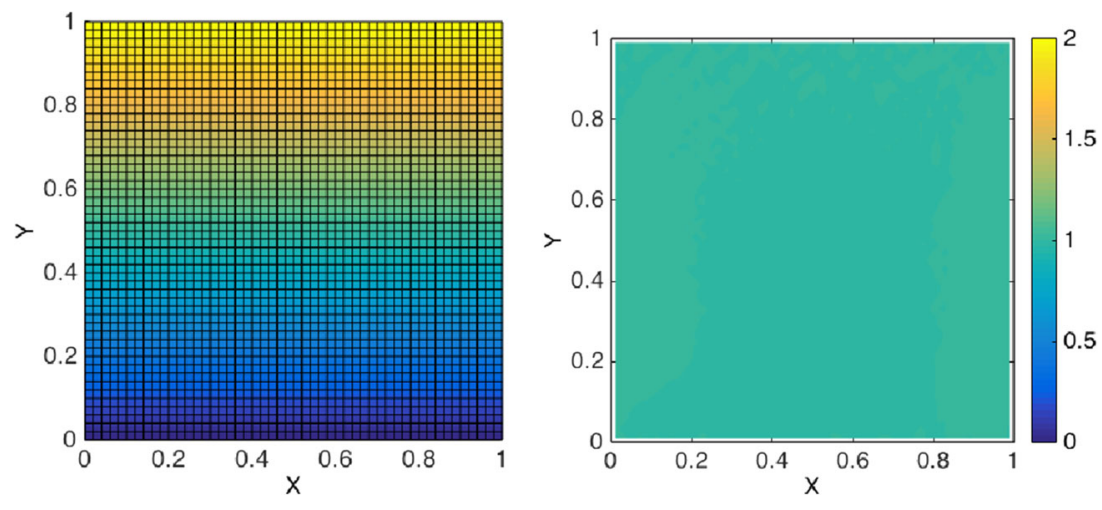


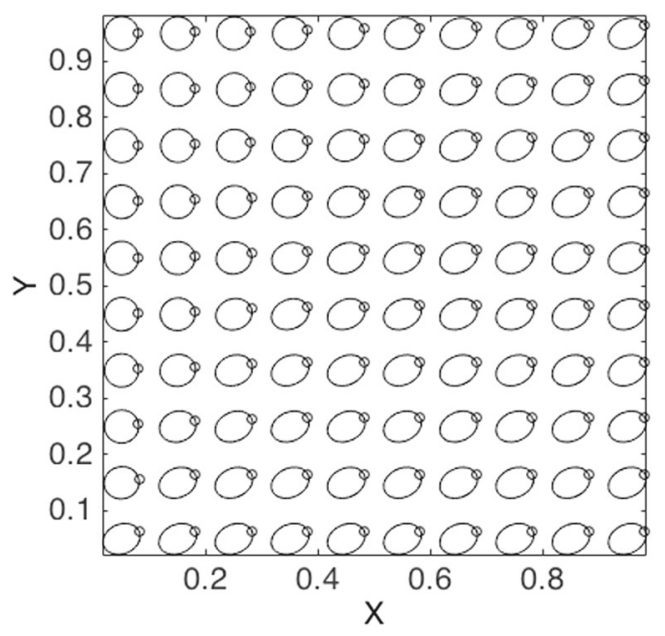

Fig. 6 Microstructure conformation in a simple shear flow

The vector $\mathbf{w}$ in Eq. 48 represents the angular velocity of the eigenvectors of the rate of strain tensor D. A simple local descriptor of the type of flow can then be constructed from the second invariant of $\mathbf{D}, \dot{\gamma}=\sqrt{2 \mathbf{D}: \mathbf{D}}$, and from the norm of $\mathbf{W},\|\mathbf{W}\|$, according to

$\chi=\frac{4\|\mathbf{W}\|}{\dot{\gamma}+2\|\mathbf{W}\|}$.

We have $0 \leq \chi \leq 2$, and more specifically

$\chi=\left\{\begin{array}{l}0 \text { in planar extension, } \\ 1 \text { in pure shear, } \\ 2 \text { in rigid motion. }\end{array}\right.$

Figure 4 shows the evolution of the microstructure conformation for different $\mathcal{K}$ in a pure shear with $\xi=$ 0.1 and $L^{0}=0.005$ (metric system of units). The qualitative analysis performed here does not require a precise determination of these coefficients. As it can be noticed, the more stiffness the spring has, the less deformation the conformation presents. In order to prove that the conformation is not only accommodating a deformation but also that it is rotating, we included a small cercle on the ellipsoid surface to appreciate the way in which that point is evolving in time.

\section{Simple shear flow}

We consider $V=2 \mathrm{~m} \cdot \mathrm{s}^{-1}$ on the N-boundary, zero velocity on the S-boundary and a linear velocity evolution on the E-boundary and W-boundaries. The microstructure effects were introduced by using $\mathcal{K}=1$.

Figure 5 depicts the velocity field, that evolves almost linearly through the domain thickness, as well as the flow criterion, that as expected corresponds to a perfect shear behaviour. The pressure field is constant in the whole flow domain.

Figure 6 shows the steady state conformation. Because of the shear, the conformation is expected to rotate clockwise. The microstructure shows a significant variation along the domain as a consequence of the different velocity and constant shear rate.

\section{Extrusion-like flow}

In the present case, we consider again $\mathcal{K}=1$. A Poiseuille velocity profile (parabolic) is enforced on the W-boundary $(-2 y(y-1), 0)^{T}$, whereas at the Eboundary the fluid leaves the square domain throughout an exit where tension-free boundary conditions are enforced.

Figure 7 depicts both components of the velocity field whereas Fig. 8 shows the steady state conformation and the flow criterion. As it can be seen, the largest conformation axis remains aligned with the streamlines. A pure extension is noticed along the symmetry axis in agreement with the flow criterion.

\section{Driven cavity flow}

The only difference with respect to the previously analyzed flow is that now a unit horizontal velocity is applied on the top wall (N-boundary) that induces the fluid flow within the cavity.

Figure 9 depicts both components of the velocity field. Figure 10 shows the conformation at a given time as
Fig. 7 Extrusion-like flow problem. Velocity field: (left) $\mathrm{x}$-component and (right) y-component
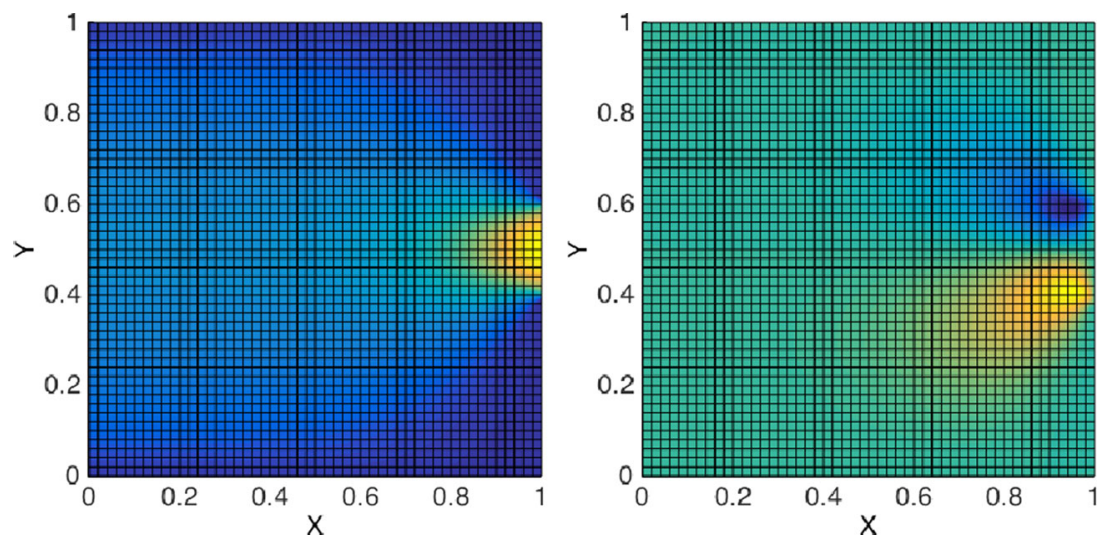
Fig. 8 Conformation distribution (left) and flow criterion (right) in the extrusion-like flow

Fig. 9 Driven cavity flow problem. Velocity field: (left) $\mathrm{x}$-components and (right) y-component

Fig. 10 Conformation (left) and flow criterion (right) in the driven cavity flow problem

Fig. 11 Flow around an obstacle. Velocity field: (left) $\mathrm{x}$-component and (right) y-components
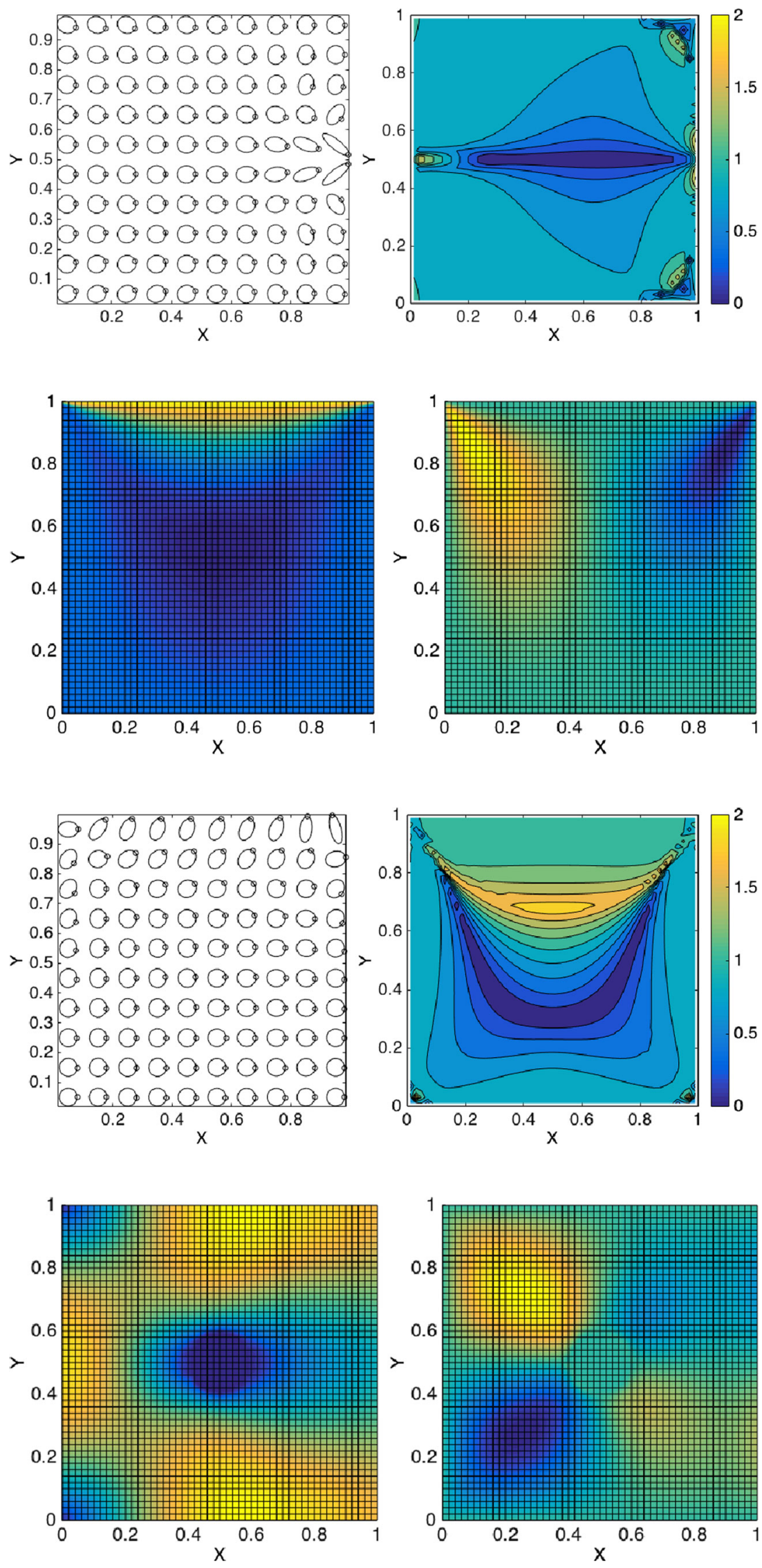
Fig. 12 Conformation (left) and flow criterion (right) in the flow around an obstacle
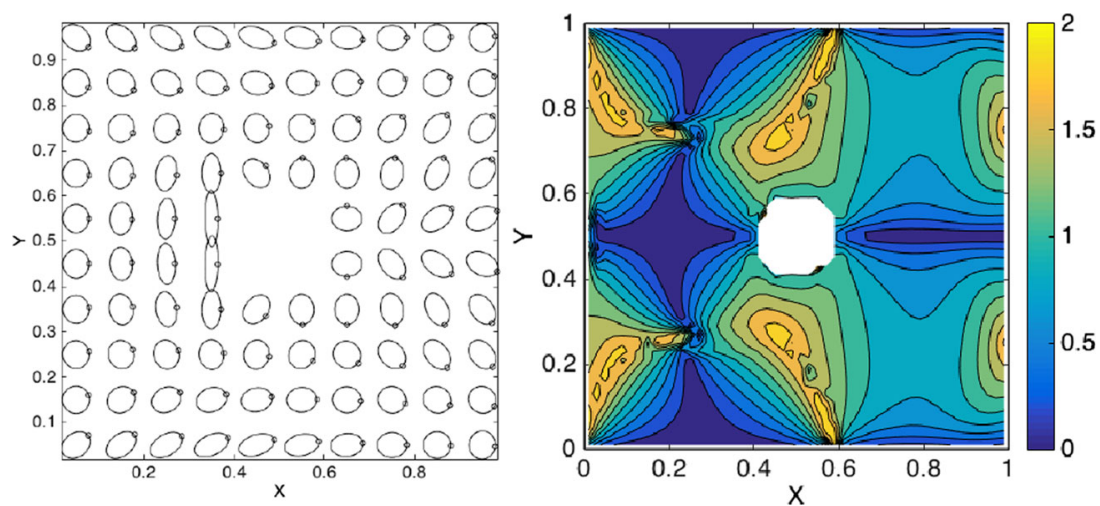

well te steady state flow crieria. The conformation evolves periodically and no steady state is reached.

\section{Flow around an obstacle}

In the present case, a unit horizontal velocity is enforced on the W-boundary, with non-slip conditions on the top and bottom walls and a free boundary condition is specified on the E-boundary. The domain contains in its center an obstacle where non-slip boundary conditions are enforced.

Figure 11 depicts both components of the velocity field. The velocity field presents two stagnation points located at the intersection between $y=0.5$ and the obstacle. These stagnation points correspond with the maximum and minimum pressures.

Figure 12 depicts the conformation and flow criteria. As it can be noticed, elongation is specially present upstream and downstream, the shear being located in the neighbourhood of the upper and lower sides of the obstacle, with the expected effects on the microstructure conformation.

\section{Coupled simulations}

This section adresses a coupled simulation in which flow kinematics induces microstructure evolution and the latter affects at its turn the flow kinematics.

Figure 13 shows two snapshots taken from films of flowing foams where the flow and microstructures were assumed almost at steady-state. The microstructure of these snapshots will be used first to identify the model parameters and then to test the agreement between the computed and observed microstructures. Of course, from the information of kinematics only, we cannot conclude on rheological aspects but at least this serves to evaluate the proposed model in terms of conformation evolution.

In order to extract from these images the conformation tensor, both images were segmented (every pixel is transformed into either black or white) and then cell boundaries were easily identified. From that, the centre of gravity of each cell can easily be obtained as well as the conformation tensor. If $\mathbf{X}_{\mathbf{i}}^{\mathbf{e}}, i=1, \ldots, Q^{e}$ are the points defining the wall of cell $\mathcal{C}^{e}$, the centre of gravity and the inertia tensor $\mathbf{J}^{e}$ are obtained from

$\mathbf{X}_{\mathbf{G}}^{\mathbf{e}}=\frac{1}{Q^{e}} \sum_{i=1}^{Q^{e}} \mathbf{X}_{\mathbf{i}}^{\mathbf{e}}$

and

$\mathbf{J}^{e}=\frac{1}{Q^{e}} \sum_{i=1}^{Q^{e}}\left(\mathbf{X}_{\mathbf{i}}^{\mathbf{e}}-\mathbf{X}_{\mathbf{G}}^{\mathbf{e}}\right) \otimes\left(\mathbf{X}_{\mathbf{i}}^{\mathbf{e}}-\mathbf{X}_{\mathbf{G}}^{\mathbf{e}}\right)$

respectively.

The numerical inertia tensor can be calculated as soon as $\mathbf{p}_{1}$ and $L_{1}$ are known ( $\mathbf{p}_{2}$ and $L_{2}$ derive from $\mathbf{p}_{1}$ and $L_{1}$ ).

$\mathbf{J}=L_{1}^{2}\left(\mathbf{p}_{1} \otimes \mathbf{p}_{1}\right)+L_{2}^{2}\left(\mathbf{p}_{2} \otimes \mathbf{p}_{2}\right)$.

In Fig. 14, the experimental ellipses related to $\mathbf{J}^{e}$ are superimposed to the cells, and also to the solution predicted

Fig. 13 Experimental snapshots (courtesy of F. Graner)

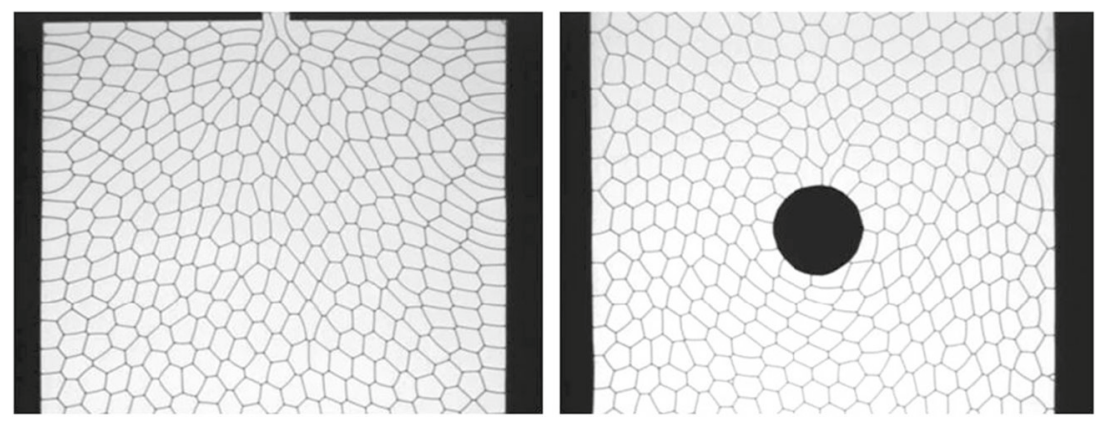


Fig. 14 Experimental microstructure with the associated conformation superimposed (left) and the associated numerical predictions (right)
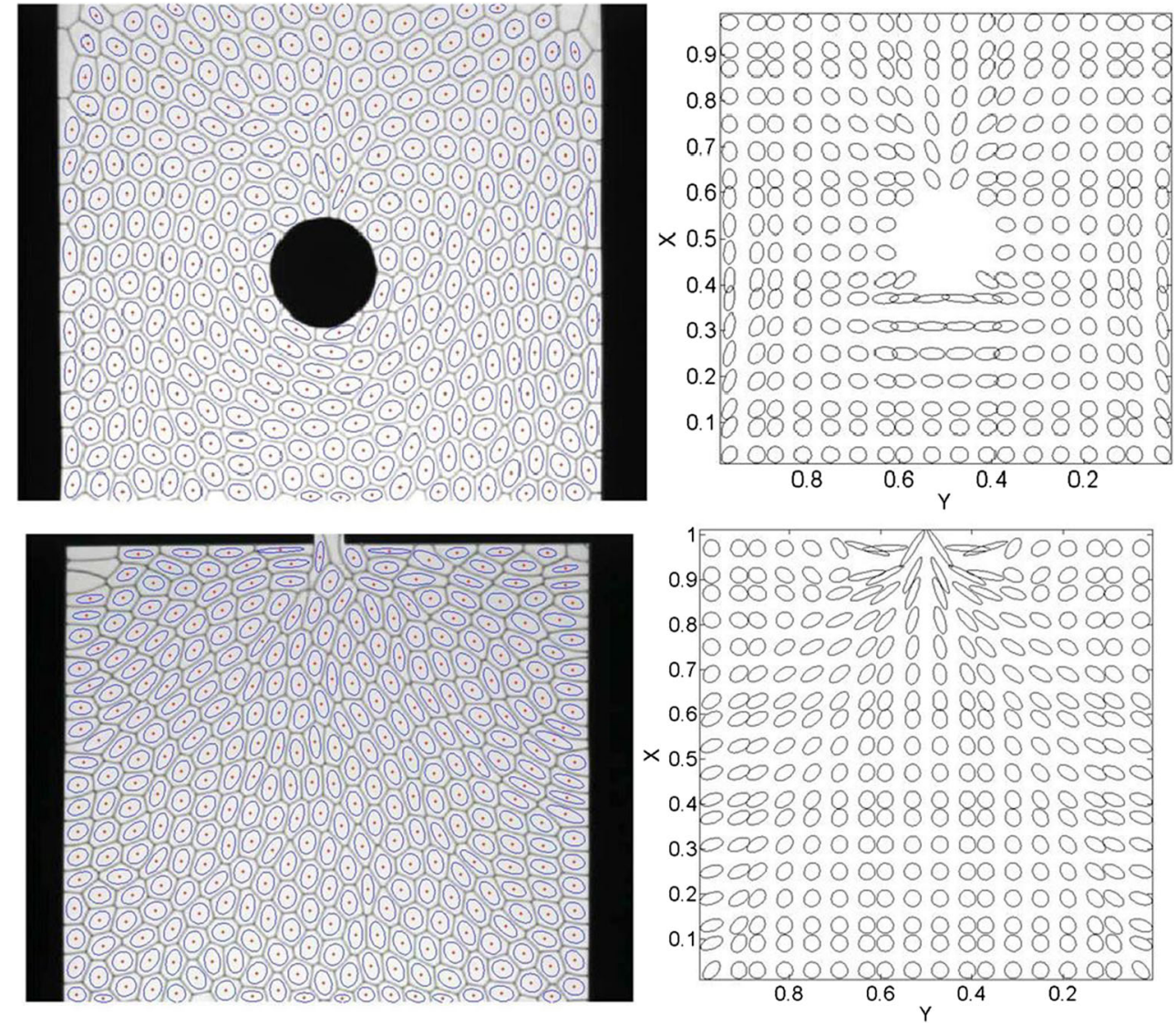

by using the proposed model. The model parameters $\mathcal{K}, \xi$, $\eta$ and $\mu$ are, as previously indicated, chosen in order to obtain cell shapes close to those observed experimentally: (metric system of units) $\mathcal{K}=1, \xi=0.1, \eta=1$ and $\mu=0.1 . L^{0}=0.005$ was identified from the average cell surface observed experimentally. In absence of rheological data, we noticed that many choices of those parameters lead to similar microstructures and that the impact of those on the kinematics was almost negligible. Thus, a proper rheological characterization seems compulsory to obtain an adequate flow-microstructure coupling.

\section{Conclusions}

This work proposes a simple model for flowing foams, where the microstructure is introduced from a conformation tensor that describes the structural elasticity. The numerical predictions have been compared with some experimental results proving the ability of the model to describe the effective kinematics as well as the flow induced microstructure evolution.

More applicative analyses, in particular in the study of industrial processes, require an appropriate rheological characterization and very probably the proposal of appropriate rheometric devices. All these aspects will be addressed in future works.

Acknowledgements This project has received funding from the European Unions Horizon 2020 research and innovation programme under the Marie Sklodowska-Curie grant agreement No. 675919.

\section{Compliance with Ethical Standards}

Conflict of interests The authors declare that they have no conflict of interest.

Publisher's Note Springer Nature remains neutral with regard to jurisdictional claims in published maps and institutional affiliations.

\section{References}

1. Benito S, Bruneau C-H, Colin T, Gay C, Molino F (2008) An elasto-visco-plastic model for immortal foams or emulsions. Eur Phys J E 25:225-251

2. Bikard J, Bruchon J, Coupez T, Vergnes B (2005) Numerical prediction of the foam structure of polymeric materials by direct 3D simulation of their expansion by chemical reaction based on a multidomain method. J Mater Sci 40/22:5875-5881

3. Binetruy C, Chinesta F, Keunings R (2015) F Flows in Polymers, Reinforced Polymers and Composites. A multiscale approach. Springer, Springerbriefs 
4. Cheddadi I, Saramito P, Raufaste C, Marmottant P, Graner F (2008) Numerical modelling of foam Couette flows. Eur Phys J E 27(/2):123-133

5. Chinesta F (2013) From single-scale to two-scales kinetic theory descriptions of rods suspensions. Arch Comput Meth Eng 20/1:129

6. Cohen-Addad S, Hohler R, Pitois O (2013) Flow in foams and flowing foams. Annu Rev Fluid Mech 45:241-267

7. Feyel F (2003) A multilevel finite element method (FE2) To describe the response of highly non-linear structures using generalized continua. Comput Methods Appl Mech Eng 192/28:32333244

8. Halin P, Lielens G, Keunings R, Legat V (1998) The Lagrangian particle method for macroscopic and micro-macro viscoelastic flow computations. J Non-Newtonian Fluid Mech 79:387-403
9. Jeffery GB (1922) The motion of ellipsoidal particles immersed in a viscous fluid. Proc R Soc London A102:161-179

10. Karimi M, Droghetti H, Marchisio DL (2017) PUFOam: a novel open-source CFD solver for the simulation of polyurethane foams. Comput Phys Commun 217:138-148

11. Keunings R (2004) Micro-macro methods for the multiscale simulation viscoelastic flow using molecular models of kinetic theory. In: Binding DM, Walters K (eds) Rheology reviews. British Society of Rheology, Aberystwyth, pp 67-98

12. Lamari H, Ammar A, Cartraud P, Legrain G, Jacquemin F, Chinesta F (2010) Routes for efficient computational homogenization of nonlinear materials using the Proper Generalized Decomposition. Arch Comput Meth Eng 17/4:373-391

13. Tlili S, Gay C, Graner F, Marcq P, Molino F, Saramito P (2015) Mechanical formalisms for tissue dynamics. Eur Phys J E 38:33-63 